\title{
Bridging unter Antikoagulation und Thrombozytenaggregationshemmung
}

Ertunc Altiok, Mohammad Almalla

\author{
Beim Bridging einer Antikoagulation wird das Blutungsrisiko - im Vergleich zum \\ thromboembolischen Risiko - oft unterschätzt. Das Bridging ist nur bei einem hohen \\ (und evtl. bei einem moderaten) thromboembolischen Risiko angezeigt. Beim Brid- \\ ging von Patienten mit der Indikation zur dualen Thrombozytenaggregationshem- \\ mung sollte eine interdisziplinäre Fallbesprechung zur Risikoabschätzung stattfin- \\ den.
}

\section{Abkürzungen}

$\begin{array}{ll}\text { aPTT } & \text { aktivierte partielle Thromboplastinzeit } \\ \text { INR } & \text { Internationale Normalisierte Ratio } \\ \text { NMH } & \text { niedermolekulares Heparin } \\ \text { NOAK } & \begin{array}{l}\text { nicht Vitamin-K-abhängige orale Antiko- } \\ \text { agulanzien }\end{array} \\ \text { OAK } & \begin{array}{l}\text { orale Antikoagulation } \\ \text { UFH }\end{array} \\ \text { unfraktioniertes Heparin } \\ \text { VKA } & \text { Vitamin-K-Antagonisten }\end{array}$

\section{Antikoagulation}

Eine orale Antikoagulation (OAK) ist meist indiziert zur Prophylaxe thromboembolischer Ereignisse bei Vorhofflimmern, zur Prävention und Behandlung tiefer Beinvenenthrombosen und Lungenarterienembolien sowie nach Herzklappenersatz. Eine Operation oder interventionelle Prozedur ist jährlich bei $10-20 \%$ aller Patienten unter OAK erforderlich [1, 2]. Die OAK wird entweder mit Vitamin-K-Antagonisten (VKA) oder mit nicht Vitamin-Kabhängigen oralen Antikoagulanzien (NOAK) durchgeführt. In Deutschland wird als VKA meist Phenprocoumon verwendet, seltener Warfarin. Es gibt 4 zugelassene NOAK,

- zum einen den direkten Thrombin-(GerinnungsfaktorIla-)Inhibitor Dabigatran,

- zum anderen die direkten Gerinnungsfaktor-Xa-Inhibitoren Rivaroxaban, Apixaban und Edoxaban.

\section{Thromboembolisches Risiko}

In der Vergangenheit wurde eine Antikoagulationstherapie in der Regel parenteral mit unfraktioniertem Heparin (UFH) oder niedermolekularem Heparin (NMH) überbrückt (engl. Bridging), solange Patienten für die periin- terventionelle Phase keine OAK erhielten. Ziel war es, thromboembolische Ereignisse zu verhindern.

In den letzten Jahren ergaben sich durch einige Studien Zweifel an der Sicherheit und Effizienz dieser BridgingStrategie. Beispielsweise erhielten in der randomisiert geplanten BRIDGE-Studie Patienten unter OAK bei Vorhofflimmern mit einem moderaten Risiko für thromboembolische Ereignisse vor einer chirurgischen Intervention randomisiert entweder NMH oder Placebo. Die Rate thromboembolischer Ereignisse war in beiden Randomisierungsarmen gleich niedrig, unter Bridging mit Heparin kam es jedoch im Vergleich zu Placebo zu einer mehr als doppelt so hohen Rate relevanter Blutungsereignisse [3]. Diese Daten weisen darauf hin, dass die Antikoagulation, im Gegensatz zu einer Bridging-Therapie, bei niedrigem bis intermediärem Risiko für thromboembolische Ereignisse pausiert werden sollte.

\section{Blutungsrisiko}

In den letzten Jahren wurde in verschiedenen Studien untersucht, ob bestimmte operative Prozeduren mit einem niedrigen Blutungsrisiko möglich sind, ohne die Antikoagulation mit VKA zu unterbrechen. Die Untersuchungen bezogen sich auf Schrittmacherimplantationen, Zahnextraktionen und Kataraktoperationen. Im Ergebnis ist es nicht nur so, dass solche Prozeduren mit niedrigem Blutungsrisiko ohne Unterbrechung der OAK durchgeführt werden können, sondern dass das Risiko für Blutungen im Operationsgebiet dabei sogar vermindert werden kann $[4,5,6]$. Dieses zunächst paradox erscheinende geringere Blutungsrisiko unter Fortführung der Antikoagulanzien erklärt sich durch die postinterventionell fehlende überlappende Gerinnungshemmung mit Heparin während der Wiederaufnahme der VKA. Daneben wird ein großer Teil der Patienten mit NOAK antikoaguliert, bei denen eine Überbrückung der pausierten Therapie mittels parenteraler Antikoagulation nicht sinnvoll ist [1]. 
- Tab.1 Zu erwartendes Blutungsrisiko bei interventionellen und operativen Eingriffen ausgewählter Fachrichtungen ohne Berücksichtigung der antithrombotischen Therapie und der individuellen Patientenfaktoren.

\begin{tabular}{|c|c|c|c|}
\hline Fachrichtung & geringes Blutungsrisiko & intermediäres Blutungsrisiko & hohes Blutungsrisiko \\
\hline Herzchirurgie & - & $\begin{array}{l}\text { Minithorakotomie, transapikaler } \\
\text { Aortenklappenersatz, Bypassopera- } \\
\text { tion, Klappenoperationen }\end{array}$ & $\begin{array}{l}\text { Reinterventionen, Endokarditis, Aor- } \\
\text { tendissektion }\end{array}$ \\
\hline Allgemeinchirurgie & $\begin{array}{l}\text { Hernioplastie, Cholezystektomie, } \\
\text { Appendektomie und Kolektomie, } \\
\text { Magenresektion, Darmresektion, } \\
\text { Brustchirurgie }\end{array}$ & $\begin{array}{l}\text { Hämorrhoidektomie, Splenektomie, } \\
\text { Gastrektomie, bariatrische Chirur- } \\
\text { gie, Rektalresektion, Thyroidekto- } \\
\text { mie }\end{array}$ & $\begin{array}{l}\text { Leberresektion, Duodenopankreat- } \\
\text { ektomie }\end{array}$ \\
\hline Gefäßchirurgie & $\begin{array}{l}\text { Endarteriektomie Karotiden, Bypass } \\
\text { oder Endarteriektomie der unteren } \\
\text { Extremitäten, endovaskuläre Angio- } \\
\text { plastie, Extremitätenamputationen }\end{array}$ & offene Bauchaortenchirurgie & $\begin{array}{l}\text { offene Thorax- oder kombinierte } \\
\text { Thorax- und Bauchchirurgie }\end{array}$ \\
\hline Orthopädie & $\begin{array}{l}\text { Handchirurgie, Schulter- und Knie- } \\
\text { arthroskopie, kleine Spinalchirurgie }\end{array}$ & $\begin{array}{l}\text { Schulterprothese, große Spinalchi- } \\
\text { rurgie, Knieoperationen, Fußchirur- } \\
\text { gie }\end{array}$ & $\begin{array}{l}\text { große Prothesenchirurgie von Knie } \\
\text { und Hüften, große Traumatologie } \\
\text { von Becken und großen Knochen, } \\
\text { proximale Femurfrakturen }\end{array}$ \\
\hline Urologie & $\begin{array}{l}\text { flexible Zystoskopie, Ureteroskopie, } \\
\text { Ureterkatheterisierung }\end{array}$ & $\begin{array}{l}\text { Prostatabiopsie, Orchiektomie, Zir- } \\
\text { kumzision }\end{array}$ & $\begin{array}{l}\text { partielle und totale Nephrektomie, } \\
\text { perkutane Nephrostomie, perkutane } \\
\text { Lithotripsie, Zystektomie und radi- } \\
\text { kale Prostatektomie, transurethrale } \\
\text { Resektion der Blase oder Prostata, } \\
\text { Penektomie, partielle Orchiektomie }\end{array}$ \\
\hline Thoraxchirurgie & $\begin{array}{l}\text { Keilresektion, diagnostische Video- } \\
\text { thorakoskopie, Brustwandresektion }\end{array}$ & $\begin{array}{l}\text { Lobektomie, Pneumoektomie, Me- } \\
\text { diastinoskopie, Sternotomie, Exzisi- } \\
\text { on mediastinaler Massen }\end{array}$ & $\begin{array}{l}\text { Ösophagektomie, Pleurapneumek- } \\
\text { tomie, Lungendekortikation }\end{array}$ \\
\hline Gastroenterologie & $\begin{array}{l}\text { Ösophago-, Gastro- und Duodeno- } \\
\text { skopie (ÖGD) ohne und mit Biopsie, } \\
\text { Endosonografie ohne Biopsie, Poly- } \\
\text { pektomie }<1 \mathrm{~cm} \text {, endoskopische } \\
\text { retrograde Cholangiopankreatogra- } \\
\text { fie (ERCP) mit ggf. Stenteinlage, Pa- } \\
\text { pillendilation ohne Sphinkterotomie }\end{array}$ & $\begin{array}{l}\text { endoskopische Feinnadelaspirati- } \\
\text { onsbiopsie solider Läsionen, Dilata- } \\
\text { tion von ösophagealen und kolorek- } \\
\text { talen Stenosen, gastroenterische } \\
\text { Stents, Argonplasma-Koagulations- } \\
\text { behandlung, Polypektomie > } 1 \mathrm{~cm} \text {, } \\
\text { perkutane endoskopische Gastros- } \\
\text { tomie (PEG), Bandligatur oder Skle- } \\
\text { rosierung bei Varizen oder Hömor- } \\
\text { rhoiden }\end{array}$ & $\begin{array}{l}\text { Dilatation bei Achalasie, Mukosekto- } \\
\text { mie/submuköse Resektion, endoso- } \\
\text { nografische Feinnadelaspirations- } \\
\text { biopsie von zystischen Läsionen des } \\
\text { Pankreas, Ampullektomie der Papilla } \\
\text { Vateri }\end{array}$ \\
\hline Gynäkologie & $\begin{array}{l}\text { diagnostische Hysteroskopie mit } \\
\text { endometrialer Biopsie, Polypekto- } \\
\text { mie, Metroplastik, Dilatation und } \\
\text { Kürettage des Uterus, zervikale Ko- } \\
\text { nisation mit Loop-Diathermie, Mar- } \\
\text { supialisation, Bartholin-Drüsen-Zys- } \\
\text { tenresektion, laparoskopische } \\
\text { Entfernung bzw. Laparotomie der } \\
\text { Adnexe bei gutartigen Erkrankun- } \\
\text { gen, Laparoskopie und Laparotomie } \\
\text { bei milder Endometriose, hystero- } \\
\text { skopische Tubensterilisation, diag- } \\
\text { nostische Laparoskopie oder mini- } \\
\text { male Operationen }\end{array}$ & $\begin{array}{l}\text { Hysteroskopie, Endometriumablati- } \\
\text { on, Laparoskopie und Laparotomie } \\
\text { bei intermediärer Endometriose, } \\
\text { einfache abdominelle und einfache } \\
\text { vaginale Hysterektomie bei gutarti- } \\
\text { gen Erkrankungen bzw. Prolaps, ra- } \\
\text { dikale Vulvektomie, Omentektomie }\end{array}$ & $\begin{array}{l}\text { Laparotomie oder laparoskopische } \\
\text { Hysterektomie bei großem Uterus, } \\
\text { laparoskopische und laparotomische } \\
\text { Myomektomie, Laparoskopie oder } \\
\text { Laparotomie bei komplizierter/tiefer } \\
\text { Endometriose, Debulking-Operation } \\
\text { bei Ovarialkarzinom, radikale Ope- } \\
\text { ration bei Zervix- und Endometri- } \\
\text { umkarzinomen, aortale Lymphaden- } \\
\text { ektomie (Becken und Lenden), } \\
\text { Beckeneviszeration }\end{array}$ \\
\hline Neurochirurgie & $\begin{array}{l}\text { Diskushernien, Laminektomie } \leq 2 \\
\text { Kanäle ohne Arthrodese, externe } \\
\text { Ventrikelableitung, intraventrikulä- } \\
\text { rer Katheterwechsel zum Druckmo- } \\
\text { nitoring, intraventrikuläre Reser- } \\
\text { voirintegration }\end{array}$ & $\begin{array}{l}\text { Laminektomie }>2 \text { Kanäle, spinale } \\
\text { Arthrodese, ventrikuloperitonealer } \\
\text { Shunt }\end{array}$ & $\begin{array}{l}\text { Entfernung intraduraler Läsionen } \\
\text { wie intrazerebraler Tumoren oder } \\
\text { intraparenchymatöser Blutungen }\end{array}$ \\
\hline
\end{tabular}


- Tab. 1 (Fortsetzung)

\begin{tabular}{|l|l|l|}
\hline Fachrichtung & geringes Blutungsrisiko & hohes Blutungsrisiko \\
\hline $\begin{array}{l}\text { interventionelle } \\
\text { Pneumologie }\end{array}$ & $\begin{array}{l}\text { Bronchoskopie zur Inspektion, } \\
\text { Bronchoaspiration, bronchoalveolä- } \\
\text { re Lavage (BAL) }\end{array}$ & $\begin{array}{l}\text { Bronchialbiopsie, transbronchiale } \\
\text { Nadelaspiration }\end{array}$ \\
\hline Ophthalmologie & $\begin{array}{l}\text { intravitrale Injektionen, Katarakt- } \\
\text { operationen, peribulbäre Anästhe- } \\
\text { sie }\end{array}$ & Vitrektomie, Trabekulektomie \\
\hline
\end{tabular}

\section{PRAXISTIPP}

Beim Bridging einer Antikoagulation wird das periinterventionelle Blutungsrisiko im Vergleich zum thromboembolischen Risiko oft unterschätzt. Bei niedrigem bis intermediärem Risiko für thromboembolische Ereignisse sollte die Antikoagulation anstatt eines Bridgings eher ganz pausiert werden. Bei Eingriffen mit einem geringen Blutungsrisiko sollte das perioperative Fortführen der Antikoagulation erwogen werden.

Zur Entscheidung über das perioperative Management der Antikoagulation kann das Blutungsrisiko anhand der Art des Eingriffs abgeschätzt werden ( $\bullet$ Tab. 1) [7].

\section{Praktische Strategie}

Bei operativen Eingriffen von Patienten unter Antikoagulation sollten folgende Aspekte beachtet werden:

- Besteht die Indikation zur Antikoagulation überhaupt noch?

- Ist eine Unterbrechung der Antikoagulation erforderlich?

- Wann soll die Antikoagulation unterbrochen werden?

- Soll - bei Entscheidung zur Unterbrechung der Antikoagulation - ein Bridging stattfinden?

- Wie soll das Bridging stattfinden?

- Wann soll die Antikoagulation wieder aufgenommen werden?

Konkret wird eine Risikoeinschätzung für das periinterventionelle Management empfohlen und entsprechend dem Eingriff und dem individuellen Risiko angepasst [1]. Wenn die Indikation zur Antikoagulation noch besteht, dann sollte geklärt werden, ob die dringende Notwendigkeit zur Durchführung eines Eingriffs mit hohem Blutungsrisiko gegeben ist ( $\boldsymbol{A}$ Abb. 1)? Um die Entscheidung für oder gegen ein Bridging treffen zu können, muss man das Blutungsrisiko gegen das individuelle Thromboembolierisiko abschätzen ( $\triangleright$ Abb. 2) [1].

\section{Vitamin-K-Antagonisten}

Patienten unter Therapie mit Vitamin-K-Antagonisten (VKA) haben ein höheres peri- und postprozedurales Blutungsrisiko. Dieses Risiko hängt wesentlich von der Art des geplanten Eingriffs ab ( $\triangleright$ Tab. 1) - ferner sind Operationen risikoreich, bei denen keine Kompression möglich ist:

- Bei Prozeduren mit hohem Blutungsrisiko sollten VKA pausiert werden.

- Bei Eingriffen mit geringem Blutungsrisiko kann die Antikoagulation fortgeführt werden. Hierzu zählen u. a. Kataraktoperationen, kleine haut- oder zahnärztliche Eingriffe. Dabei ist es sinnvoll, für die INR (Internationale Normalisierte Ratio) einen zum Operationszeitpunkt niedrigen therapeutischen Bereich anzustreben.

Substanzen aus der Gruppe der VKA sind Phenprocoumon, Acenocoumarol (keine Zulassung in Deutschland) und Warfarin. In Deutschland wird meist Phenprocoumon und seltener Warfarin verschrieben. Der hauptsächliche Unterschied zwischen den Wirkstoffen liegt in den Halbwertszeiten: Phenprocoumon hat mit 72-270 Stunden die längste Halbwertszeit, Acenocoumarol mit 8-24 Stunden die kürzeste und Warfarin liegt mit 36-42 Stunden dazwischen.

Die INR kann durch eine Reihe von Interaktionen mit oft verordneten Medikamenten beeinflusst werden:

- Die Wirkung der VKA wird beispielsweise durch nicht steroidale Antirheumatika, Allopurinol und Levothyroxin verstärkt.

- Die Wirkung der VKA wird durch Rifampicin, Azathioprin, Carbamazepin und Glukokortikoide abgeschwächt.

Zusätzlich sind Wechselwirkungen durch die Ernährung zu berücksichtigen. Die therapeutische Breite von VKA ist gering, sodass bereits geringe Änderungen der Dosis dazu führen können, dass der angestrebte INR-Zielkorridor nicht erreicht wird.

In der Regel können Operationen bei einer INR unter 1,5 sicher durchgeführt werden. Eine Unterbrechung der An- 


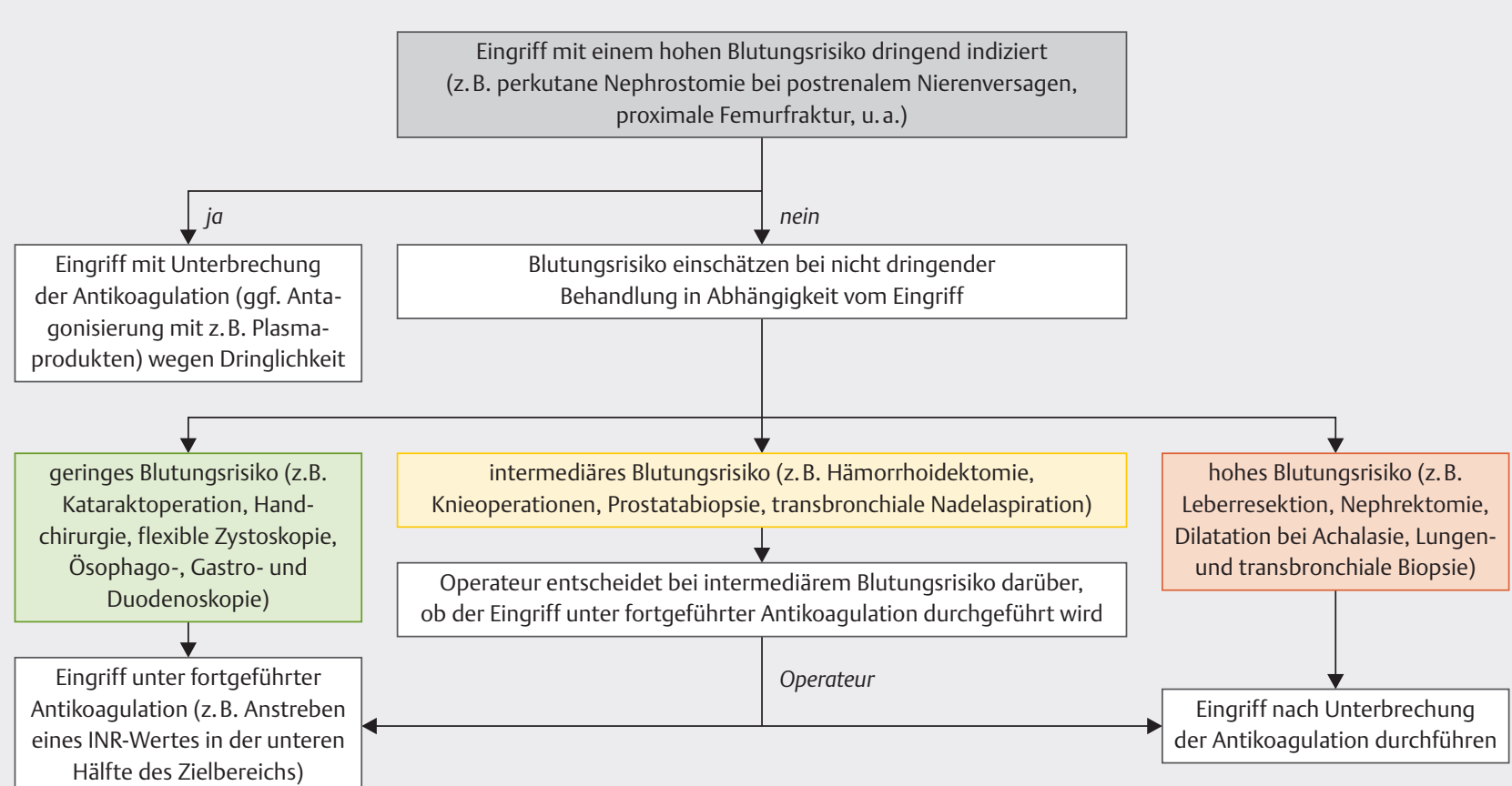

- Abb. 1 Algorithmus für die prozedurale Unterbrechung der Antikoagulation.
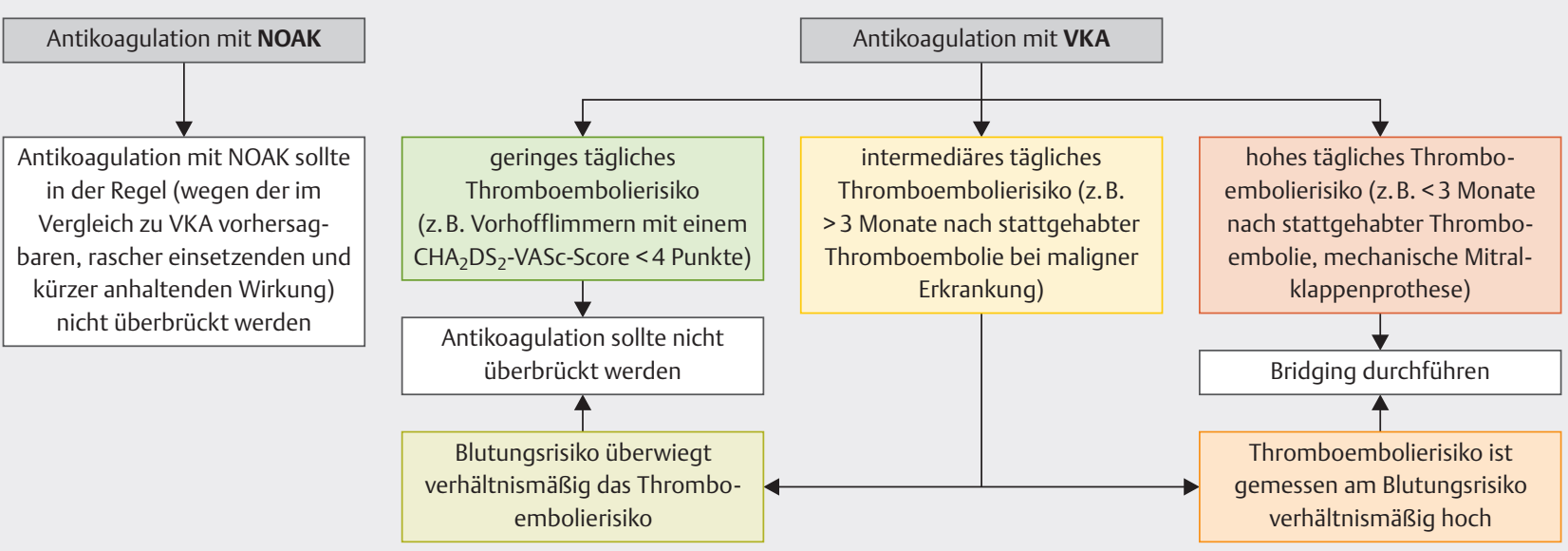

- Abb.2 Algorithmus für das Bridging der Antikoagulation; NOAK= nicht Vitamin-K-abhängige orale Antikoagulanzien, VKA=Vitamin-K-Antagonisten.

tikoagulation kann bei Hochrisikopatienten bewirken, dass Thromboembolien auftreten. Als Hochrisikopatienten gelten u. a.:

- Patienten mit Vorhofflimmern bei einem $\mathrm{CHA}_{2} \mathrm{DS}_{2}-$ VASc-Score von $\geq 4$ Punkten

- Patienten mit mechanischen Herzklappen oder weniger als 3 Monate zurückliegender Mitralklappenrekonstruktion

- Patienten mit kürzlich zurückliegendem thromboembolischen Ereignis
- Patienten mit besonderen schweren Formen einer Thrombophilie [8]

Wenn ein Bridging von VKA als sinnvoll erachtet wird, dann sollte man das NMH dem UFH vorziehen. VKA sollten 3-5 Tage vor dem Eingriff pausiert werden mit dann täglichen INR-Kontrollen, bis die INR unter 1,5 liegt.

Mit NMH oder UFH beginnt man, wenn die INR unter 2 ist. Bei hohem Thromboembolierisiko wird dabei die zweimal tägliche, gewichtsadaptierte Gabe von NMH empfohlen. 
Eine Gerinnungskontrolle des NMH ist nicht erforderlich, wenn man die Nierenfunktion berücksichtigt. Bei Niereninsuffizienz ist der Einsatz des $\mathrm{NMH}$ im Gegensatz zum UFH eingeschränkt. NMH sollte spätestens 12 Stunden vor dem Eingriff zum letzten Mal appliziert werden. Am Operationstag sollte man die INR kontrollieren.

NMH oder UFH sollte postoperativ frühestens nach 6-12 Stunden in der vor der Operation verordneten Dosis wieder verabreicht werden - bei hohem postoperativen Blutungsrisiko wird die erneute Heparintherapie auf bis zu 48-72 Stunden nach dem Eingriff verschoben, wobei dann 6-8 Stunden nach der Operation eine Thromboseprophylaxe mit am besten subkutanem $\mathrm{NMH}$ verordnet wird.

Die Medikation mit VKA sollte am ersten oder zweiten postoperativen Tag begonnen werden: An den ersten beiden Tagen sollte die Dosis 50 \% über der bisherigen Erhaltungsdosis liegen. NMH oder UFH sollte so lange gegeben werden, bis die angestrebte therapeutische INR erreicht wird [8].

Bei Überdosierung mit VKA ist eine Therapie mit einem spezifischen Antagonisten möglich: Vitamin $\mathrm{K}_{1}$ (Phytomenadion) ist in der Lage, die antikoagulatorische Wirkung innerhalb von 24 Stunden aufzuheben. Bei leichteren Blutungen ist eine Antagonisierung meist jedoch nicht notwendig und es genügt, die VKA-Dosis vorübergehend zu verringern. Durch die Antagonisierung mit Vitamin $\mathrm{K} 1$ wird eine effektive Antikoagulation für mehrere Tage verhindert. Wenn man in Fällen von sehr starker oder bedrohlicher Blutung den Eintritt der vollen Vitamin- $\mathrm{K}_{1}$-Wirkung nicht abwarten kann, gibt man Prothrombinkomplex-Konzentrat (PPSB) oder frisch gefrorenes Plasma (FFP) und kann damit die VKA-Wirkung unmittelbar aufheben. Die Elimination von VKA kann man zusätzlich beschleunigen, indem man Colestyramin oral verabreicht und dadurch den enterohepatischen Kreislauf unterbricht.

\section{Heparin}

Heparin-Präparate werden entweder unfraktioniert als UFH oder fraktioniert als NMH angewendet:

- Heparin bindet an verschiedene Antithrombinmoleküle, vor allem Antithrombin III und wirkt dadurch antikoagulatorisch.

- NMH, das durch Spaltung und Fraktionierung der niedermolekularen Anteile aus konventionellem Heparin gewonnen wird, hemmt überwiegend selektiv den Gerinnungsfaktor Xa. Als NMH stehen in Deutschland neben der Leitsubstanz Enoxaparin noch Certoparin, Dalteparin, Nadroparin, Reviparin und Tinzaparin zur Verfügung.
Als Nebenwirkung kann sowohl beim UFH als auch beim $\mathrm{NMH}$ eine heparininduzierte Thrombozytopenie auftreten. Dieses Risiko ist mit einer Rate von weniger als $1 \%$ beim NMH geringer als beim UFH. Für die Kontrollen gilt:

- Im Gegensatz zum UFH ist eine Gerinnungskontrolle unter NMH in der Regel nicht erforderlich, eine Therapiekontrolle ist jedoch durch die Bestimmung der Anti-Faktor-Xa-Aktivität möglich.

- Unter UFH ist eine Therapiekontrolle durch Bestimmung der aktivierten partiellen Thromboplastinzeit (aPTT) erforderlich: Als Ziel gilt eine 1,5-2,5-fache Verlängerung der aPTT.

Der Vorteil des NMH liegt darin, dass es subkutan appliziert werden kann, während eine kontrollierte therapeutische Antikoagulation mit UFH lediglich intravenös möglich ist. Da NMH überwiegend renal eliminiert wird, ist eine Einschränkung bei Niereninsuffizienz zu beachten. $\mathrm{NMH}$ wird subkutan gewichtsadaptiert in therapeutischer Dosis und zur Thromboseprophylaxe in verringerter Dosis gegeben. Die Halbwertszeit von subkutan verabreichtem $\mathrm{NMH}$ beträgt etwa 4 Stunden, sodass es vor Operationen letztmals spätestens 12 Stunden vor dem Eingriff gegeben werden sollte - bei besonders hohem operativen Blutungsrisiko 24 Stunden vor dem Eingriff. Intravenös verabreichtes UFH hat eine Halbwertszeit von 60 bis 90 Minuten, d.h., dass die antikoagulatorische Wirkung nach 3-4 Stunden Pause vergeht [9].

Als Antidot für UFH kann Protamin eingesetzt werden. Wegen der relativ kurzen Wirkdauer von UFH ist dies jedoch selten erforderlich. Das Antidot Andexanet alfa wurde zur Anwendung bei Patienten, die mit Faktor-Xa-Inhibitoren behandelt werden, entwickelt - diese Substanz wurde jedoch nicht für den Einsatz als Antidot gegen $\mathrm{NMH}$ zugelassen. Eine Zulassung für $\mathrm{NMH}$ besteht nicht; eine Wirkung des Antidot ist jedoch wegen des Wirkmechanismus zu erwarten. Die Zulassung besteht nur für die Substanzen Apixaban und Rivaroxaban.

\section{Nicht Vitamin-K-abhängige orale Antikoagu- lanzien}

Nicht Vitamin-K-abhängige orale Antikoagulanzien (NOAK) werden als Alternative zu den VKA zur therapeutischen Antikoagulation für die Indikationen nicht valvuläres Vorhofflimmern und venöse Thromboembolie eingesetzt. Von den 4 verschiedenen NOAKs ist Dabigatran ein direkter Thrombin-Inhibitor, und Rivaroxaban, Apixaban und Edoxaban sind Faktor-Xa-Inhibitoren. Wegen der im Vergleich zu VKA relativ kurzen Eliminationshalbwertszeiten von je nach Substanz etwa 9-17 Stunden ist in der Regel kein Bridging mit NMH oder UFH erforderlich: Man spricht von einem gut definierten „On“- und „Off“-Effekt der NOAK. 
Bei Patienten mit eingeschränkter Nierenfunktion ist Folgendes zu beachten:

- Dabigatran wird mit einem Anteil von 80 \% überwiegend renal eliminiert.

- Bei Rivaroxaban, Apixaban und Edoxaban überwiegt eine nicht renale Ausscheidung (der Anteil der renalen Ausscheidung dieser Substanzen liegt zwischen 27 und $50 \%)$.

Dabigatran ist dementsprechend bei einer GFR <30 ml/ min nicht zugelassen. Rivaroxaban, Apixaban und Edoxaban dürfen prinzipiell bei eingeschränkter Nierenfunktion bis zu einer GFR von $15 \mathrm{ml} / \mathrm{min}$ verordnet werden. In den relevanten Studien zu allen 4 NOAKs wurden jedoch nur Patienten mit einer GFR $\geq 30 \mathrm{ml} / \mathrm{min}$ eingeschlossen (Ausnahme: Apixaban $\geq 25 \mathrm{ml} / \mathrm{min}$ oder Kreatinin $<2,5 \mathrm{mg} / \mathrm{dl}$ ), sodass die Datenlage nur aussagekräftig ist, solange die Einschränkung der Nierenfunktion moderat bleibt [10].

Bei Patienten mit Lebererkrankungen gilt es bei der OAK abzuwägen: Patienten mit Lebererkrankungen haben sowohl ein erhöhtes Risiko für thromboembolische Ereignisse als auch ein erhöhtes Blutungsrisiko. Es besteht die falsche Annahme, dass bei fortgeschrittener Leberinsuffizienz automatisch durch ein gewisses Maß an Antikoagulation ein Schutz vor Thromboembolien besteht. Dazu ist der teilweise nicht unerhebliche hepatische Metabolismus der NOAK bei Patienten mit Lebererkrankungen zu beachten: Die hepatische Ausscheidung von Dabigatran ist unter den NOAK mit einem Anteil von $20 \%$ am ge- ringsten. In den Zulassungsstudien aller 4 NOAK zeigte sich kein Unterschied in Bezug auf hepatische Nebenwirkungen zwischen NOAK und VKA: Die Inzidenz hepatischer Nebenwirkungen war bei den in den Studien zugelassenen Patientengruppen insgesamt sehr niedrig, wobei Patienten mit relevanter Leberinsuffizienz in den Studien ausgeschlossen wurden.

Bei Patienten mit Lebererkrankungen gilt: Im Child-PughStadium A dürfen alle NOAK verordnet werden, im ChildPugh-Stadium B dürfen Dabigatran und Apixaban verordnet werden, wohingegen im Child-Pugh-Stadium $C$ keines der NOAK zugelassen ist - VKA sind unter INR-Kontrollen prinzipiell in allen Child-Pugh-Stadien zugelassen $(\triangleright$ Abb. 3) [11].

Präoperativ sollte genau festgelegt werden, wann das NOAK zum letzten Mal vor dem Eingriff einzunehmen ist - dabei spielen die Nierenfunktion und das operative Blutungsrisiko eine Rolle ( $\triangleright$ Abb.4) $[8,10]$.

Die NOAK-Therapie wird nach Eingriffen mit sofortiger und kompletter Blutstillung nach 6-12 Stunden wieder aufgenommen. Hier ist der im Vergleich zu den VKA bereits genannte rasche Wirkungseintritt nach Einnahme zu beachten. Bei Prozeduren mit hohem Blutungsrisiko wird eine Wiederaufnahme der NOAK-Therapie erst nach bis zu 48-72 Stunden empfohlen, wobei dann ab 6 Stunden nach einer Operation eine Thromboseprophylaxe, z. B. mit subkutan verabreichtem NMH in verringerter Dosierung, gegeben wird: Hier entscheidet die postoperative
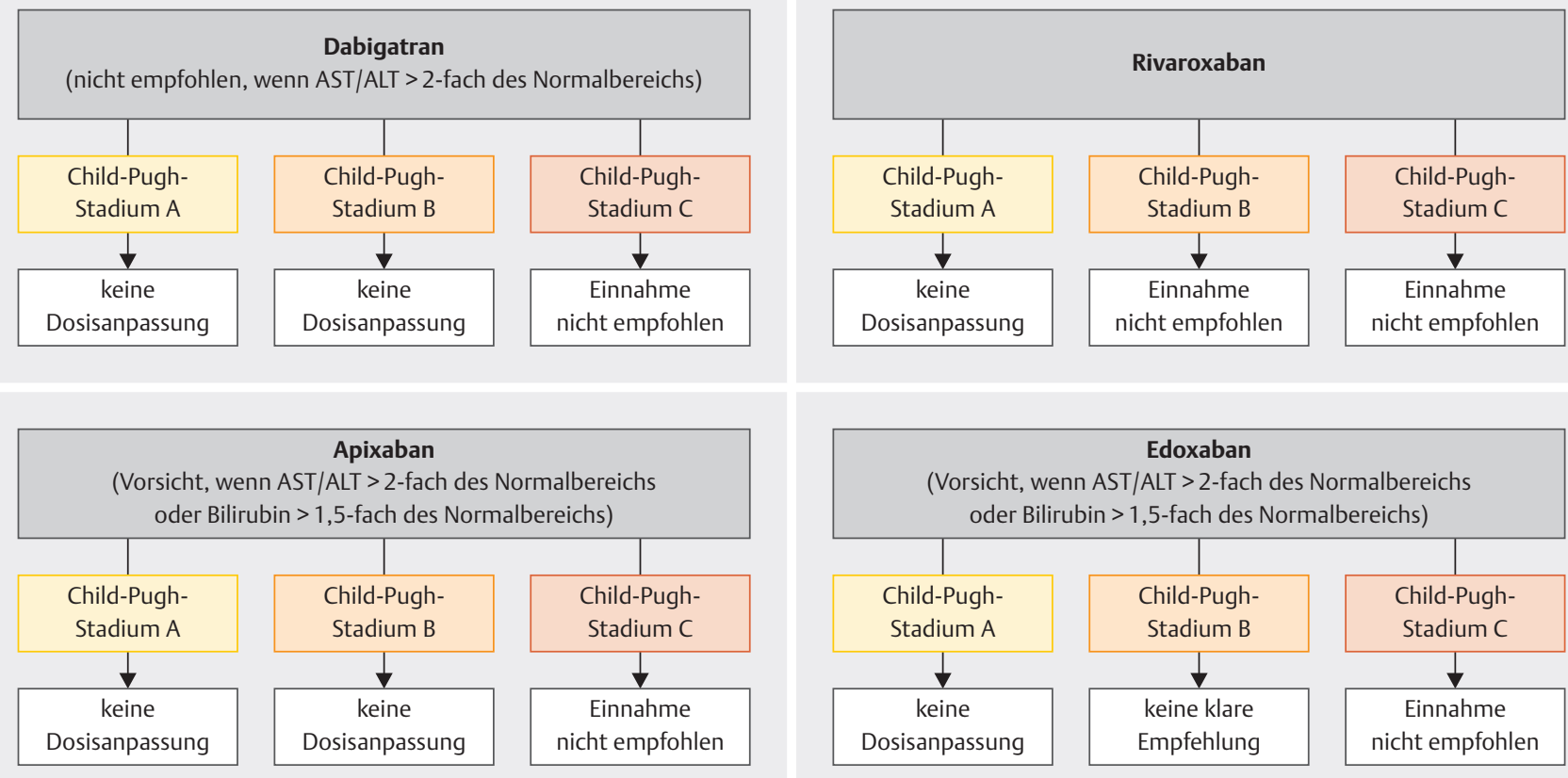

- Abb. 3 Schema zur Einnahme von nicht Vitamin-K-abhängigen oralen Antikoagulanzien (NOAK) bei Lebererkrankungen unter Berücksichtigung des Child-Pugh-Scores zur Stadieneinteilung der Leberzirrhose; ALT = Alaninaminotransferase, AST = Aspartataminotransferase. 


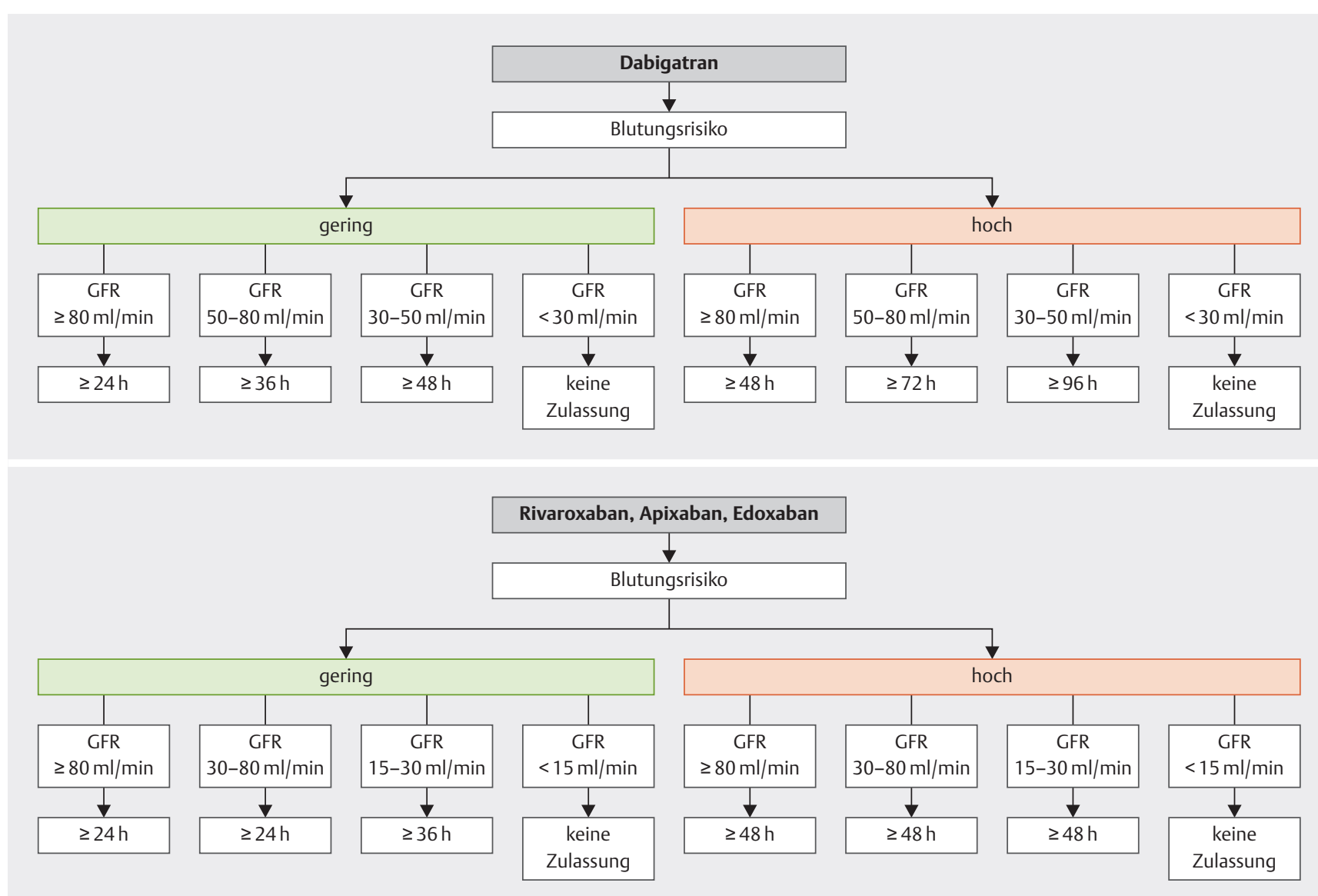

- Abb.4 Schema zur letzten Einnahme von nicht Vitamin-K-abhängigen oralen Antikoagulanzien (NOAK) vor einemelektiven operativen Eingriff in Abhängigkeit vom Blutungsrisiko und der Nierenfunktion, gemessen an der glomerulären Filtrationsrate (GFR).

Blutungsneigung, wann die therapeutische Antikoagulation wieder aufgenommen wird [10].

\section{PRAXISTIPP}

Eine Antikoagulation mit NOAK sollte wegen des im Vergleich zu VKA raschen Wirkungsein- und -austritts nicht überbrückt werden.

Nur selten besteht eine Indikation zur spezifischen Antagonisierung der Gerinnungshemmung mit NOAK: Meist genügen allgemeine Maßnahmen der Hämostase und das Abwarten des Wirkungsaustritts. Für den Fall, dass die antikoagulatorische Wirkung rasch aufgehoben werden muss, wie bei Notfalloperationen und bei lebensbedrohlichen oder nicht beherrschbaren Blutungen, wurde für den direkten Thrombininhibitor Dabigatran ein spezifisches Antidot entwickelt: Idarucizumab hebt die antikoagulatorische Wirkung von Dabigatran rasch und sicher auf [12].
Zur Anwendung bei Patienten, die mit Faktor-Xa-Inhibitoren behandelt werden, wurde das Antidot Andexanet alfa entwickelt: Es bindet direkte Faktor-Xa-Inhibitoren mit der gleichen Affinität wie der natürliche Gerinnungsfaktor Xa; dadurch wird die gerinnungshemmende Wirkung der Faktor-Xa-Inhibitoren aufgehoben. Für Apixaban und Rivaroxaban besteht eine Zulassung; für NMH und Edoxabsan besteht keine Zulassung. Jedoch ist zu beachten, dass es bei $10 \%$ der mit dem Antidot behandelten Patienten zu einem thromboembolischen Ereignis kam [13].

Bei lebensbedrohlichen Blutungen unter Therapie mit Edoxaban, die mit Maßnahmen wie z. B. Transfusion oder Hämostase nicht beherrscht werden können, wird die Gabe eines Prothrombinkomplex-Konzentrates (PPSB) in einer Dosierung von $50 \mathrm{IE} / \mathrm{kg}$ empfohlen. PPSB kann die Wirkungen von Edoxaban 30 Minuten nach Ende der Infusion aufheben. 
- Tab. 2 Parameter des $\mathrm{CHA}_{2} \mathrm{DS}_{2}$-VASc-Scores.

\begin{tabular}{|c|c|c|}
\hline $\begin{array}{l}\text { Buch- } \\
\text { stabe }\end{array}$ & Parameter & Punkte \\
\hline C & Herzinsuffizienz („congestive heart failure“) & 1 \\
\hline $\mathrm{H}$ & Hypertonie & 1 \\
\hline A & Alter > 74 Jahre & 2 \\
\hline D & Diabetes mellitus & 1 \\
\hline$S$ & Schlaganfall & 2 \\
\hline V & $\begin{array}{l}\text { vaskuläre Erkrankung im Sinne einer periphe- } \\
\text { ren arteriellen Verschlusskrankheit, eines vo- } \\
\text { rangegangenen Herzinfarkts oder komplexer } \\
\text { Aortenplaques }\end{array}$ & 1 \\
\hline A & Alter 65-74 Jahre & 1 \\
\hline Sc & weibliches Geschlecht („sex category“) & 1 \\
\hline
\end{tabular}

\section{Bridging in besonderen Situationen}

\section{Vorhofflimmern}

Das Thromboembolierisiko bei nicht valvulärem Vorhofflimmern wird mit dem $\mathrm{CHA}_{2} \mathrm{DS}_{2}$-VASc-Score abgeschätzt. Der $\mathrm{CHA}_{2} \mathrm{DS}_{2}$-VASc-Score beinhaltet die in - Tab. 2 Faktoren und Punkte. Es kann eine maximale Punktzahl von 9 erreicht werden - mit zunehmender Zahl steigt die Rate thromboembolischer Ereignisse: Bei $\geq 4$ Punkten spricht man von einem hohen Risiko.

Somit sollte bei operativen Eingriffen mit einem hohen Blutungsrisiko bei einem $\mathrm{CHA}_{2} \mathrm{DS}_{2}$-VASc-Score von $\geq 4$ Punkten ein Bridging erwogen werden [8]. Außerdem besteht ein hohes Thromboembolierisiko innerhalb von 3 Monaten nach einem Schlaganfall bzw. transitorisch ischämischer Attacke und bei valvulärem Vorhofflimmern. Letzteres ist definiert als Vorhofflimmern bei mittelschwerer oder schwerer Mitralklappenstenose bzw. bei mechanischen Herzklappenprothesen. Bei valvulärem Vorhofflimmern besteht unabhängig vom $\mathrm{CHA}_{2} \mathrm{DS}_{2}$ VASc-Score ein besonders hohes Risiko für Thromboembolien [1].

\section{PRAXISTIPP}

Ein Vorhofflimmern bei einem $\mathrm{CHA}_{2} \mathrm{DS}_{2}$-VASc-Score von $\geq 4$ Punkten oder ein valvuläres Vorhofflimmern gehen mit einem erhöhten Risiko für Thromboembolien einher. Unter valvulär versteht man ein Vorhofflimmern bei mittelschwerer oder schwerer Mitralklappenstenose bzw. bei mechanischen Herzklappenprothesen.

\section{FALLBEISPIEL}

\section{Antikoagulation mit VKA bei Vorhofflimmern}

Eine 78-jährige Patientin wird wegen einer symptomatischen Bradykardie stationär aufgenommen. Bei symptomatischen Pausen bis 6 Sekunden in der Monitorüberwachung wird die Indikation zur Schrittmacherimplantation gestellt. Es besteht eine orale Antikoagulation mit Phenprocoumon bei langjährig bekanntem permanentem Vorhofflimmern. Ein Schrittmacher kann komplikationslos unter Fortführung der Antikoagulation mit einer im niedrigen therapeutischen Korridor eingestellten INR von 2,0 bis 2,5 implantiert werden.

\section{Venöse Thromboembolien}

In den ersten 3 Monaten nach einer tiefen Beinvenenthrombose und/oder Lungenarterienembolie gilt: Es besteht ein hohes perioperatives Thromboembolierisiko. Daher sollten elektive Eingriffe nach Möglichkeit bis 3 Monate nach der Thrombose/Embolie verschoben werden. Wenn ein dringlicher operativer Eingriff in diesen ersten 3 Monaten erforderlich wird, dann ist unter Antikoagulation mit VKA ein Bridging indiziert. Sollte ein dringender operativer Eingriff mit hohem Blutungsrisiko gar innerhalb eines Monats nach einer venösen Thromboembolie erforderlich werden, dann sollte bei vorhandener Expertise zumindest die präoperative Implantation eines Vena-cava-Filters erwogen werden [9].

Außerdem besteht ein hohes Thromboembolierisiko bei Patienten mit nachgewiesener schwerer Thrombophilie, hierzu gehören u.a.: der Protein-C-, der Protein-S- oder der Antithrombinmangel und das Antiphospholipidsyndrom [1].

\section{CAVE}

In den ersten 3 Monaten nach einer tiefen Beinvenenthrombose und/oder Lungenarterienembolie sollten elektive Eingriffe vermieden werden.

\section{FALLBEISPIEL}

Antikoagulation mit NOAK bei venöser Thromboembolie

Eine 63-jährige Patientin stellt sich in der Allgemeinchirurgie vor zur elektiven laparoskopischen Cholezystektomie bei seit Jahren bestehender symptomatischer Cholezystolithiasis. Es besteht - seit einem Rezidiv einer tiefen Beinvenenthrombose vor 2 Monaten - eine dauerhafte Antikoagulation mit Rivaroxaban. 
Es wird die Entscheidung getroffen, den Eingriff um 1 Monat zu verschieben, um ein Zeitintervall von mindestens 3 Monaten nach der Beinvenenthrombose einzuhalten. Bei normaler Nierenfunktion mit einer glomerulären Filtrationsrate von $>80 \mathrm{ml} / \mathrm{min}$ wird vereinbart, Rivaroxaban letztmalig 24 Stunden vor dem Operationstermin einzunehmen.

\section{Mechanische Herzklappenprothesen}

Bei mechanischen Herzklappenprothesen sind lediglich VKA zur OAK zugelassen. Das Management zur Antikoagulation während nicht kardialer Eingriffe sollte bei diesen Patienten präoperativ geplant werden. Auch hier gilt, die Antikoagulation während kleinerer Operationen, bei denen eine Blutung leicht zu kontrollieren ist, nicht zu unterbrechen. Wurde die Entscheidung zugunsten eines Bridgings getroffen, sollte die INR - nach Pausieren der VKA - für größere chirurgische Maßnahmen < 1,5 betragen; eine Überbrückung mit Heparin wird empfohlen.

UFH ist die einzige zugelassene Heparintherapie für Patienten mit mechanischen Prothesen, die intravenöse Gabe wird bevorzugt. Die Verwendung von subkutanem $\mathrm{NMH}$ ist eine „Off-Label“-Alternative zum UFH für das Bridging: Wird NMH eingesetzt, dann sollte es zweimal täglich in an das Körpergewicht angepasster therapeutischer Dosierung verabreicht werden - wenn möglich, unter laborchemischer Kontrolle der Anti-Xa-Aktivität mit einem Ziel-Wert von 0,5-1,0 U/ml [14].

\section{Thrombozytenaggregationshemmung}

Innerhalb von 5 Jahren nach koronarer Stentimplantation findet bei bis zu einem Viertel dieser Patienten ein nicht kardialer operativer Eingriff statt [8]. Bei Patienten mit geplanter Operation unter dualer Plättchenhemmung ist

- das Risiko einer Stentthrombose,

- die Konsequenzen einer Verzögerung des Eingriffs und

- das erhöhte perioperative Blutungsrisiko

zu berücksichtigen. Wegen der Komplexität ist eine interdisziplinäre Diskussion ratsam, um das thrombotische Risiko und das Blutungsrisiko gegeneinander abzuwägen [15].

Das perioperative Risiko für kardiale Ereignisse kann in 3 Gruppen unterteilt werden ( $\bullet$ Tab. 3) [8]:

- niedriges Risiko (Rate eines kardialen Todes oder

Myokardinfarkts innerhalb von 30 Tagen < $1 \%$ )

- intermediäres Risiko (Rate von 1-5\%)

- hohes Risiko (Rate $\geq 5 \%$ )
- Tab. 3 Einschätzung des Risikos eines kardialen Todes oder Myokardinfarkts innerhalb von 30 Tagen: geringes Risiko $<1 \%$, intermediäres Risiko $1-5 \%$ und hohes Risiko $\geq 5 \%$.

\begin{tabular}{|c|c|}
\hline $\begin{array}{l}\text { Risiko- } \\
\text { gruppe }\end{array}$ & Eingriffe \\
\hline gering & $\begin{array}{l}\text { oberflächliche Chirurgie, Brust, Zahn, Schilddrüse, Auge, } \\
\text { asymptomatische Karotiden mittels Stenting oder Endar- } \\
\text { teriektomie, kleine gynäkologische Eingriffe, kleine ortho- } \\
\text { pädische Eingriffe wie Meniskusoperation, kleine urologi- } \\
\text { sche Eingriffe }\end{array}$ \\
\hline $\begin{array}{l}\text { interme- } \\
\text { diär }\end{array}$ & $\begin{array}{l}\text { intraperitoneal wie Splenektomie, Hiatushernien und Cho- } \\
\text { lezystektomien, symptomatische Karotiden mittels Sten- } \\
\text { ting oder Endarteriektomie, periphere arterielle Angioplas- } \\
\text { tie, endovaskuläre Angioplastie bei Aneurysmen, Kopf- und } \\
\text { Halschirurgie, große neurologische und orthopädische } \\
\text { Operationen wie Spinal- und Hüftchirurgie, große urologi- } \\
\text { sche Operationen, Nierentransplantation, kleine intratho- } \\
\text { rakale Eingriffe }\end{array}$ \\
\hline hoch & $\begin{array}{l}\text { Chirurgie der Aorta und großen Gefäße, offene Operation } \\
\text { der unteren Extremitäten zur Revaskularisierung, Amputa- } \\
\text { tion oder Thromboembolektomie, Chirurgie an Duodenum } \\
\text { und Pankreas, Leberresektion und Gallengangsoperation, } \\
\text { Ösophagektomie, Chirurgie bei Darmperforation, vollstän- } \\
\text { dige Zystektomie, Pneumektomie, Lungen- und Leber- } \\
\text { transplantation }\end{array}$ \\
\hline
\end{tabular}

\section{Plättchenhemmung}

\section{Medikamente}

Eine duale Plättchenhemmung ist in der Regel zeitweise nach einer Stentimplantation zur Prävention einer Stentthrombose erforderlich. Dabei wird Azetylsalizylsäure in Kombination mit einem der folgenden P2Y12-Rezeptorantagonisten gegeben: Clopidogrel, Ticagrelor oder Prasugrel. Clopidogrel und Prasugrel wirken genauso wie Azetylsalizylsäure irreversibel für die gesamte Lebensdauer der Thrombozyten von etwa 5 bis 12 Tagen. Erst neu gebildete Thrombozyten sind voll funktionsfähig. Demgegenüber wirkt Ticagrelor reversibel, d. h. die Thrombozyten werden mit dem fallenden Medikamentenspiegel wieder funktionsfähig. Ticagrelor wird zweimal täglich eingenommen.

\section{Unterbrechung der Medikation}

Wird die Entscheidung getroffen, die duale Plättchenhemmung zu unterbrechen, dann sollte die folgende Mindestdauer vom Absetzen der Medikation mit dem P2Y12-Rezeptorantagonisten bis zum operativen Eingriff eingehalten werden:

- Ticagrelor mindestens 3 Tage

- Clopidogrel mindestens 5 Tage

- Prasugrel mindestens 7 Tage

Die duale Plättchenhemmung sollte postoperativ, so früh wie von operativer Seite her möglich, für die anfangs fest- 
gelegte Dauer fortgeführt werden: In der Regel kann die duale Plättchenhemmung 1-4 Tage nach dem operativen Eingriff wieder aufgenommen werden [15].

\section{PRAXISTIPP}

Wird die duale Plättchenhemmung ausgesetzt, sollten nach dem Pausieren der P2Y12-Rezeptorantagonisten bei Ticagrelor mindestens 3 Tage, bei Clopidogrel mindestens 5 Tage und bei Prasugrel mindestens 7 Tage bis zum Eingriff vergehen.

\section{Generelle Strategie}

Ist eine duale Plättchenhemmung indiziert, sollte der entsprechende Fall vor einem geplanten operativen Eingriff nach Möglichkeit interdisziplinär besprochen werden, um das Risiko des Patienten einschätzen zu können [15]. Bei den meisten operativen Eingriffen wird das Fortführen der Medikation mit Azetylsalizylsäure empfohlen, weil der Nutzen die negativen Effekte in der Regel überwiegt, insbesondere wenn die Entscheidung getroffen wird, die duale Plättchenhemmung vorzeitig zu unterbrechen - Ausnahmen sind z. B. Operationen im Spinalbereich oder neurochirurgische Eingriffe. Generell sollten operative Eingriffe innerhalb des ersten Monats nach koronarer Stentimplantation vermieden werden [8].

\section{PRAXISTIPP}

Bei Patienten mit Indikation für Azetylsalizylsäure wird das Fortführen dieser Medikation meist unabhängig von der Strategie empfohlen: Ausnahmen sind z. B. Operationen im Spinalbereich oder neurochirurgische Eingriffe.

Sollte ein dringender operativer Eingriff geplant werden, für den eine notwendige duale Plättchenhemmung unterbrochen werden muss, sollte dieser mindestens auf 1 Monat nach koronarer Stentimplantation verschoben werden - dies gilt sowohl nach Einsetzen von unbeschichteten Stents (BMS) als auch beschichteten Stents (DES). Bei Patienten nach einem Myokardinfarkt oder mit hohem ischämischen Risiko sollten operative Eingriffe wenn möglich auf bis zu 6 Monate nach dem Ereignis bzw. nach Stentimplantation verschoben werden. Faktoren, die ein hohes ischämisches Risiko anzeigen, sind:

- eine vorherige Stentthrombose

- ein Stent in dem einzig offen verbliebenen Koronargefäß

- eine diffuse Mehrgefäßerkrankung insbesondere bei Diabetes mellitus

- chronische Niereninsuffizienz
- mindestens 3 implantierte Stents oder behandelte Läsionen

- Bifurkation mit 2 implantierten Stents

- totale Stentlänge $>60 \mathrm{~mm}$

- Rekanalisierung eines zuvor chronisch verschlossenen Koronargefäßes [15]

\section{CAVE}

Elektive operative Eingriffe sollten innerhalb von 1 Monat nach koronarer Stentimplantation generell vermieden werden. Bei akutem Koronarsyndrom oder hohem ischämischen Risiko sollten elektive Eingriffe nach Möglichkeit bis zu 6 Monate nach dem Ereignis bzw. der Stentimplantation verschoben werden.

In den seltenen Fällen, in denen ein operativer Eingriff innerhalb der Zeit von weniger als 1 Monat nach koronarer Stentimplantation durchgeführt und beide Thrombozytenaggregationshemmer pausiert werden müssen, gilt: Eine Bridging-Strategie mit kurz wirksamen Thrombozytenaggregationshemmern (Cangrelor, Tirofiban oder Eptifibatid) kann erwogen werden [15]. Cangrelor ist ein intravenös zu verabreichender reversibler P2Y12Rezeptorantagonist (die zuvor genannten P2Y12-Rezeptorantagonisten Clopidogrel, Ticagrelor und Prasugrel werden oral verabreicht): Die Wirkung setzt innerhalb von Minuten ein und muss durch eine kontinuierliche Infusion aufrechterhalten werden. Wegen der im Vergleich zu den übrigen P2Y12-Rezeptorantagonisten sehr kurzen Halbwertszeit von wenigen Minuten ist die vollständige Thrombozytenfunktion innerhalb von einer Stunde nach Beendigung der Infusion wiederhergestellt. Demgegenüber stehen Tirofiban und Eptifibatid als Inhibitoren des Glykoprotein-Ilb/IIla-Rezeptors, welche die Thrombozytenfunktion besonders effektiv hemmen. Tirofiban und Eptifibatid werden intravenös verabreicht. Die Wirkung setzt ebenfalls rasch ein - die Halbwertszeit beider Substanzen beträgt etwa 2 bzw. 2,5 Stunden. In einem solch besonderen Fall, in dem diese Bridging-Strategie erwogen wird, wird empfohlen: Der Eingriff sollte in einem Zentrum durchgeführt werden, in dem eine durchgehende Herzkatheterbereitschaft bei Auftreten einer Stentthrombose besteht [8].

\section{FALLBEISPIEL}

Duale Plättchenhemmung nach koronarer Stentimplantation

Ein 57-jähriger Patient mit ausgeprägtem kardiovaskulären Risikoprofil wird wegen thorakaler Beschwerden stationär in die Kardiologie eingewiesen. Ein akuter Myokardinfarkt kann ausgeschlossen werden. Weil die nicht invasive Bildgebung eine belastungsabhängige Ischämie der Hinterwand zeigt, wird eine 
Koronarangiografie durchgeführt. In dieser wird eine hochgradige Stenose der rechten Koronararterie diagnostiziert und ein beschichteter Stent (DES) implantiert. Eine duale Plättchenhemmung mit Azetylsalizylsäure und Clopidogrel wird für 6 Monate empfohlen.

Im weiteren Aufenthalt werden in der Thoraxröntgenaufnahme und in der anschließenden Computertomografie der Zufallsbefund einer Raumforderung gestellt, die suspekt auf ein Bronchialkarzinom ist. Es wird die Entscheidung getroffen, den Patienten zunächst zu entlassen und nach 1 Monat wieder einzubestellen zur dann dringlichen diagnostischen Bronchoskopie mit ggf. transbronchialer Biopsie; hierzu wird Clopidogrel 1 Monat nach Stentimplantation für 5 Tage abgesetzt und die Medikation mit Azetylsalizylsäure während des endoskopischen Eingriffs unverändert fortgeführt.

\section{KERNAUSSAGEN}

- Eine orale Antikoagulation sollte nicht für Eingriffe mit geringem Blutungsrisiko unterbrochen werden.

- Ein Bridging der oralen Antikoagulation sollte in der Regel Patienten unter Vitamin-K-Antagonisten (VKA) und einem hohen Risiko für thromboembolische Ereignisse vorbehalten bleiben.

- Entsprechend sollte während der perioperativen Pause der Antikoagulation kein Bridging stattfinden, wenn ein geringes oder intermediäres Thromboembolierisiko vorliegt.

- Generell sollten nicht Vitamin-K-abhängige orale Antikoagulanzien (NOAK) nicht gebridgt werden.

- In den ersten 3 Monaten nach stattgehabter venöser Thromboembolie sollten elektive Eingriffe vermieden werden.

- Eine duale Plättchenhemmung sollte im ersten Monat nach elektiver koronarer Stentimplantation nach Möglichkeit generell nicht unterbrochen werden.

- Nach akutem Koronarsyndrom sollten elektive Eingriffe nach Möglichkeit erst nach 6 Monaten stattfinden.

\section{Zitierweise für diesen Artikel}

Phlebologie 2022; 51: 37-50. DOI: 10.1055/a-16790677

Dieser Beitrag ist eine aktualisierte Version des Artikels: Altiok E, Almalla M. Bridging unter Antikoagulation und Thrombozytenaggregationshemmung. Kardiologie up2date 2020;16: 243 - 256 .

\section{Wissenschaftlich verantwortlich gemäß Zerti- fizierungsbestimmungen}

Dr. med. Ertunc Altiok, Aachen

\section{Interessenkonflikt}

\section{Erklärung zu finanziellen Interessen}

Forschungsförderung erhalten: nein; Honorar/geldwerten Vorteil für Referententätigkeit erhalten: ja, von einer anderen Institution (Pharma- oder Medizintechnikfirma usw.); Bezahlter Berater/interner Schulungsreferent/Gehaltsempfänger: nein; Patent/Geschäftsanteile/Aktien (Autor/Partner, Ehepartner, Kinder) an im Bereich der Medizin aktiven Firma: nein; Patent/Geschäftsanteile/Aktien (Autor/Partner, Ehepartner, Kinder) an zu Sponsoren dieser Fortbildung bzw. durch die Fortbildung in ihren Geschäftsinteressen berührten Firma: nein

Erklärung zu nichtfinanziellen Interessen

Mitgliedschaft bei der Deutschen Gesellschaft für Kardiologie (DGK) und bei der Europäischen Gesellschaft für Kardiologie (ESC).

\section{Autorinnen/Autoren}

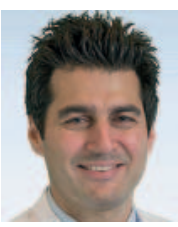

\section{Ertunc Altiok}

Priv.-Doz. Dr. med. 1995-2001 Medizinstudium in Aachen. 2003 Promotion. 2014 Venia Legendi für das Fach „Innere Medizin“. Facharzt für Innere Medizin - Kardiologie, Internistische Intensivmedizin, Interventionelle Kardiologie. Seit 2016 Oberarzt in der Medizinischen Klinik 1 der Uniklinik Aachen. Leiter Sektion III: nicht invasive und Hybrid-Bildgebung.

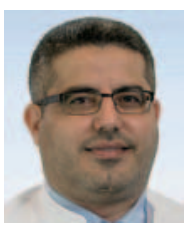

\section{Mohammad Almalla}

Dr. med. 1993-2000 Medizinstudium in Aleppo, Syrien. Facharzt für Innere Medizin - Kardiologie, Interventionelle Kardiologie. Seit 2016 Oberarzt in der Medizinischen Klinik 1 der Uniklinik Aachen. Stellvertretender Leiter Sektion I: interventionelle Klappentherapie und konven-

tionelle interventionelle Kardiologie.

\section{Korrespondenzadresse}

PD Dr. med. Ertunc Altiok

Uniklinik RWTH Aachen, Klinik für Kardiologie, Angiologie und Internistische Intensivmedizin

Pauwelsstraße 30

52074 Aachen

Deutschland

ealtiok@ukaachen.de

\section{Literatur}

[1] Rechenmacher SJ, Fang JC. Bridging Anticoagulation: Primum Non Nocere. J Am Coll Cardiol 2015; 66: 1392-1403. doi:10.1016/j.jacc.2015.08.002

[2] Lange CM, Fichtlscherer S, Miesbach W et al. The Periprocedural Management of Anticoagulation and Platelet Aggregation 
Inhibitors in Endoscopic Interventions. Dtsch Arztebl Int 2016; 113: 129-135. doi:10.3238/arztebl.2016.0129

[3] Douketis JD, Spyropoulos AC, Kaatz S et al. Perioperative Bridging Anticoagulation in Patients with Atrial Fibrillation. N Engl ] Med 2015; 373: 823-833. doi:10.1056/NEJMoa1501035

[4] Birnie DH, Healey JS, Wells GA et al. Pacemaker or defibrillator surgery without interruption of anticoagulation. N Engl ] Med 2013; 368: 2084-2093. doi:10.1056/NEJMoa1302946

[5] Bajkin BV, Popovic SL, Selakovic SD. Randomized, prospective trial comparing bridging therapy using low-molecular-weight heparin with maintenance of oral anticoagulation during extraction of teeth. J Oral Maxillofac Surg 2009; 67: 990-995. doi:10.1016/j.joms.2008.12.027

[6] Schlitt A, Jambor C, Spannagl M et al. The perioperative management of treatment with anticoagulants and platelet aggregation inhibitors. Dtsch Arztebl Int 2013; 110: 525-532. doi:10.3238/arztebl.2013.0525

[7] Rossini R, Musumeci G, Visconti LO et al. Perioperative management of antiplatelet therapy in patients with coronary stents undergoing cardiac and non-cardiac surgery: a consensus document from Italian cardiological, surgical and anaesthesiological societies. Eurolntervention 2014; 10: 38-46. doi:10.4244/EIJV10I1A8

[8] Kristensen SD, Knuuti J, Saraste A et al. 2014 ESC/ESA Guidelines on non-cardiac surgery: cardiovascular assessment and management: The Joint Task Force on non-cardiac surgery: cardiovascular assessment and management of the European Society of Cardiology (ESC) and the European Society of Anaesthesiology (ESA). Eur Heart J 2014; 35: 2383-2431. doi:10.1093/eurheartj/ehu282

[9] Baron TH, Kamath PS, McBane RD. Management of antithrombotic therapy in patients undergoing invasive procedures. N Engl J Med 2013; 368: 2113-2124. doi:10.1056/ NEJMra1206531
[10] Heidbuchel H, Verhamme P, Alings M et al. Updated European Heart Rhythm Association practical guide on the use of nonvitamin-K antagonist anticoagulants in patients with non-valvular atrial fibrillation: Executive summary. Eur Heart J 2017; 38: 2137-2149. doi:10.1093/eurheartj/ehw058

[11] Qamar A, Vaduganathan M, Greenberger NJ et al. Oral Anticoagulation in Patients With Liver Disease. J Am Coll Cardiol 2018; 71: 2162-2175. doi:10.1016/j.jacc.2018.03.023

[12] Pollack CV jr., Reilly PA, van Ryn J et al. Idarucizumab for Dabigatran Reversal - Full Cohort Analysis. N Engl J Med 2017; 377 : 431-441. doi:10.1056/NEJMoa1707278

[13] Connolly S], Crowther M, Eikelboom JW et al. Full Study Report of Andexanet Alfa for Bleeding Associated with Factor Xa Inhibitors. N Engl J Med 2019; 380: 1326-1335. doi:10.1056/ NEJMoa1814051

[14] Baumgartner H, Falk V, Bax |] et al. 2017 ESC/EACTS Guidelines for the management of valvular heart disease. Eur Heart ] 2017; 38: 2739-2791. doi:10.1093/eurheartj/ehx391

[15] Valgimigli M, Bueno H, Byrne RA et al. 2017 ESC focused update on dual antiplatelet therapy in coronary artery disease developed in collaboration with EACTS: The Task Force for dual antiplatelet therapy in coronary artery disease of the European Society of Cardiology (ESC) and of the European Association for Cardio-Thoracic Surgery (EACTS). Eur Heart J 2018; 39: 213-260. doi:10.1093/eurheartj/ehx419

Bibliografie

Phlebologie 2022; 51: 37-50

DOI 10.1055/a-1679-0677

ISSN 0939-978X

(C) 2022. Thieme. All rights reserved.

Georg Thieme Verlag KG, Rüdigerstraße 14,

70469 Stuttgart, Germany 


\section{Punkte sammeln auf CME.thieme.de}

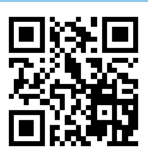

Diese Fortbildungseinheit ist in der Regel 12 Monate online für die Teilnahme verfügbar.

Unter https://eref.thieme.de/CXIU8UJ oder über den QR-Code kommen Sie direkt zur Startseite des Wissenstests und zum Artikel. Sie finden dort auch den genauen Einsendeschluss.

Sollten Sie Fragen zur Online-Teilnahme haben, finden Sie unter https://cme.thieme.de/hilfe eine ausführliche Anleitung.

Wir wünschen viel Erfolg beim Beantworten der Fragen!

VNR 2760512022161723350

\section{Frage 1}

Nur eine der folgenden Aussagen zum Bridging ist richtig. Welche?

A Bei Eingriffen mit geringem Blutungsrisiko sollte eine Antikoagulation normalerweise unterbrochen werden.

B Bei hohem thromboembolischen Risiko sollten Vitamin- K-Antagonisten (VKA) nicht gebridgt werden.

C Das Blutungsrisiko wird oft unterschätzt.

D Ein Bridging von nicht Vitamin-K-abhängigen oralen Antikoagulanzien (NOAK) sollte in der Regel durchgeführt werden.

E Ein Bridging von Vitamin-K-Antagonisten (VKA) ist immer indiziert.

\section{Frage 2}

Welche der folgenden Substanzen ist ein Vitamin-K-Antagonist $(\mathrm{VKA})$ ?
A Dabigatran
B Rivaroxaban
C Apixaban
D Edoxaban
E Phenprocoumon

\section{Frage 3}

Nur einer der genannten Eingriffe hat ein geringes Blutungsrisiko. Welcher?
A Dekortikation der Lunge
B Prostatabiopsie
C Hämorrhoidektomie
D Thyroidektomie
E Appendektomie

\section{Frage 4}

Wodurch sind Vitamin-K-Antagonisten (VKA) gekennzeichnet?

A Phenprocoumon, Acenocoumarol und Warfarin sind in Deutschland zugelassen.

B Warfarin hat eine längere Halbwertszeit als Phenprocoumon.

C Die Wirkung der VKA wird durch Glukokortikoide abgeschwächt.
D Mit dem Bridging von VKA mit niedermolekularem (NMH) oder unfraktioniertem Heparin (UFH) wird begonnen, sobald die INR unter 1,5 liegt.

E Die Wirksamkeit von VKA ist unabhängig von der Nahrungsaufnahme des Patienten.

\section{Frage 5}

Wie viele Stunden muss die letzte Einnahme von Rivaroxaban vor einem elektiven operativen Eingriff bei geringem Blutungsrisiko und einer glomerulären Filtrationsrate (GFR) von $20 \mathrm{ml} / \mathrm{min}$ zurückliegen?
$A \geq 24 h$
B $\geq 36 h$
$\mathrm{C} \geq 48 \mathrm{~h}$
$\mathrm{D} \geq 72 \mathrm{~h}$
$E \geq 96 h$

\section{Frage 6}

Welcher der folgenden Parameter geht in den $\mathrm{CHA}_{2} \mathrm{DS}_{2}$-VAScScore ein?
A Diabetes mellitus
B männliches Geschlecht
C Niereninsuffizienz
D tiefe Beinvenenthrombose in der Anamnese
$\mathrm{E}$ erhöhte Leberenzyme

\section{Frage 7}

Bei welchem Eingriff besteht ein hohes Risiko ( $\geq 5 \%$ ) eines kardialen Todes oder Myokardinfarkts innerhalb von 30 Tagen?
A Cholezystektomie
B Hiatushernienchirurgie
C Meniskus-OP
D Nierentransplantation
E Pankreaschirurgie

\section{- Weitere Fragen auf der folgenden Seite...}




\section{Punkte sammeln auf CME.thieme.de}

\section{Frage 8}

Wie viel Tage nach Absetzen von Clopidogrel kann ein elektiver operativer Eingriff durchgeführt werden?
A nach mindestens 3 Tagen
B nach mindestens 4 Tagen
$C$ nach mindestens 5 Tagen
D nach mindestens 6 Tagen
E nach mindestens 7 Tagen

\section{Frage 9}

Wie viel Tage nach einem operativen Eingriff kann die Therapie mit Clopidogrel in der Regel wieder fortgesetzt werden?
A sofort
B nach 1-4 Tagen
C nach 3-5 Tagen
D nach 7 Tagen
E nach 14 Tagen

\section{Frage 10}

Nur eine der folgenden Aussagen zu mechanischen Herzklappenprothesen ist richtig. Welche?

A Bei Entscheidung zugunsten eines Bridgings bei mechanischen Herzklappenprothesen kann nach Pausieren des Vitamin- KAntagonisten (VKA) ein operativer Eingriff bei einer INR zwischen 1,5 und 2 sicher durchgeführt werden.

B Bei mechanischen Herzklappenprothesen sind sowohl Vitamin- K-Antagonisten (VKA) als auch nicht Vitamin-K-abhängige orale Antikoagulanzien (NOAK) zur Antikoagulation zugelassen.

C Bei mechanischen Herzklappenprothesen sollte die Antikoagulation auch für kleinere Operationen, bei denen eine Blutung zu kontrollieren ist, unterbrochen werden.

D Niedermolekulares Heparin (NMH) ist für ein Bridging als „OffLabel“-Therapie keine Alternative zu unfraktioniertem Heparin (UFH).

E Unfraktioniertes Heparin (UFH) ist die einzige zugelassene Heparintherapie für Patienten mit mechanischen Herzklappenprothesen. 